\title{
COMPUTATIONS OF WAKEFIELDS IN THE ILC COLLIMATORS*
}

\author{
J. D. A. Smith, Cockcroft Institute/Lancaster University, Daresbury, UK ${ }^{\dagger}$ \\ C. J. Glasman, Cockcroft Institute/Manchester University, Daresbury, UK
}

\begin{abstract}
The collimators in the ILC serve the dual purpose of reducing the beam halo and as of a form of machine protection from potentially miss-steered beams. However, there is a significant wakefield in the immediate vicinity of the beam caused by their presence. It is important to be able to predict this short-range wake-field and the extent which it dilutes the emittance of the beam. We extend the previous analysis [1], [2] of wake-fields in collimators to realistic short bunches applicable to the ILC. We achieve these results using the finite difference code GdfidL. The angular wake is decomposed into its constituent components for rectangular collimators and compared with their circular collimator counterparts. Comparisons are made between these simulations, existing analytical models, and experimental results.
\end{abstract}

\section{INTRODUCTION}

There have been 16 prototype collimators tested at End Station A at SLAC so far. Preliminary results for the first eight were shown last year. Improvements in GdfidL [3], and availability of time on a 54 node cluster [4] have allowed the refinement of the simulations, and results are available for comparison with other simulation codes. In addition, simulations have been carried out on the collimators tested in March 2007, which are described separately in these proceedings [5]. There are only five different geometries present in these more recent runs, the first of which was also tested last year. Results are available for both longitudinal and transverse kick factors. As the transverse kicks are comparable with current experiments, we shall discuss these in this paper. This paper will also discuss estimates of the errors in the kick factors for these collimators.

\section{ESTIMATION OF KICK FACTORS}

Each of the collimators that are being considered have been simulated with a a few offsets of the beam from the axis of the collimator towards the jaws, different bunch lengths ranging from the ILC bunch length of $\sigma_{z}=0.3 \mathrm{~mm}$ to in some cases up to $5 \mathrm{~mm}$ in order to achieve fast results and observe the overall trend, and varying mesh settings. The convention chosen was to have the aperture vary in $\mathrm{y}$, with $\mathrm{x}$ fixed as the beam pipe size. Consequently somewhat less mesh was required in $\mathrm{x}$ than in $\mathrm{y}$, and we choose the

\footnotetext{
* This work is supported by the Commission of the European Communities under the 6th Framework Programme "Structuring the European Research Area”, contract number RIDS-011899

$\dagger$ j.d.smith@lancs.ac.uk

05 Beam Dynamics and Electromagnetic Fields
}

resolution in y to be roughly homogenous with z, ensuring there is plenty of mesh around the beam region. The mesh resolution is characterised by the number of cells per $\sigma_{z}$ of the bunch. A number of results for a given collimator with the same offset and bunch length are plotted together. In most cases, the 'real' value is determined by fitting the points with a curve and extrapolating to the infinite mesh point. It is expected that the accuracy improves with the $\log$ of the typical cell dimensions, so an exponential curve is fitted to the data as shown in Figure 1.

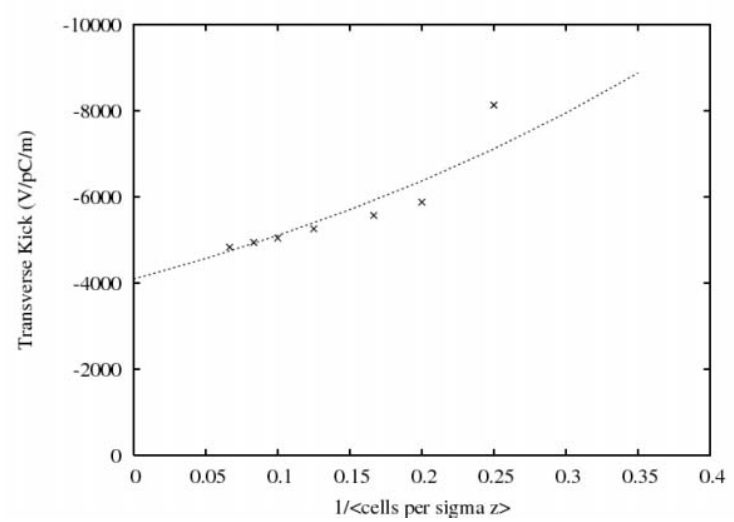

Figure 1: Variation of GdfidL calculated kick for collimator 3 (depth $1 \mathrm{~m}$ ) for $\sigma_{z}=1.0 \mathrm{~mm}$ at an offset of $0.4 \mathrm{~mm}$ with mesh resolution

The error can be estimated from the quality of the fit, although with no inherent error in the calculations and only estimates of systematic effects such as the non conformal nature of the grid and numerical dispersion this may not be a good reflection of the uncertainty. Alternatively a more conservative estimate of error can be made from the difference between the infinite mesh point and the highest resolution GdfidL calculation value that we have.

Each result is then combined with others for the same collimator and bunch length with different offsets, and transverse loss factor is plotted, with the error derived from the previous plot. Figure 2 shows this, with the green error bar the difference between highest resolution GdfidL results and the infinite mesh extrapolation.

Results can then be compared for different collimators and bunch lengths, as the kick is the gradient of the linear part of the plot of loss factor against beam offset.

\section{SLAC 2006 COLLIMATORS}

The moving mesh algorithm in GdfidL and the availability of the escience cluster [4] have allowed improvements to be made to the simulations of collimators and presented

D04 Instabilities - Processes, Impedances, Countermeasures 


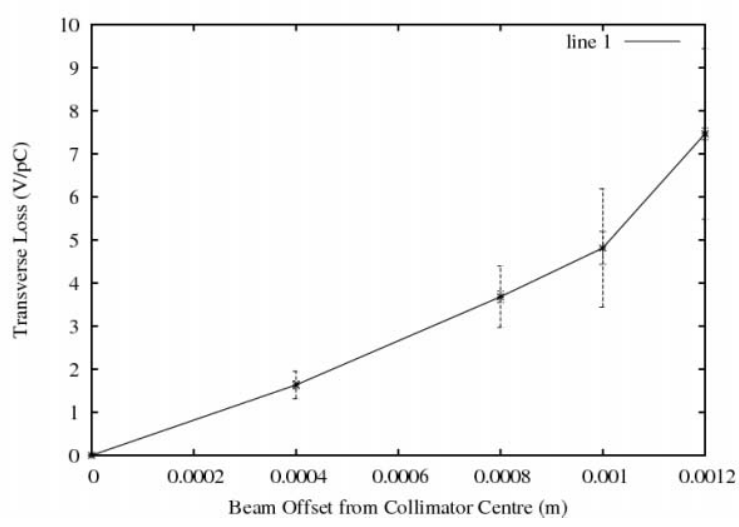

Figure 2: GdfidL calculated kick for collimator 3 (depth $1 \mathrm{~m}$ ) for $\sigma_{z}=1.0 \mathrm{~mm}: \kappa_{\perp}=4.09 \pm 0.80 \mathrm{~V} / \mathrm{pC} / \mathrm{mm}$

last year. The descriptions and justifications of the choice of collimator geometries are given in reference [6]. The conservative estimate of uncertainty described previously can be used. A summary of the transverse kick factors is presented in Table 1 .

These can be compared with calculations using ECHO3D by Zagorodnov [7], and are in good general agreement. The errors on the collimators 2 and 3 (marked *) suggest these warrant further work, and the step tapers 4 and 5 were such that the mesh had to be handled in a slightly different way, so the estimate of error was taken from term to term values where these were available (marked $\ddagger$ ), and where this was not possible, the calculated value is presented without error (marked $\dagger$ ).

\section{SLAC 2007 COLLIMATORS}

There were in fact only five distinct collimator geometries for the March 07 run at SLAC end station A. Transverse kick factors for these geometries are displayed in Table 2 This would appear to suggest good wakefield performance can be expected from the exponential profile taper. The calculated value for Collimator 15 at $0.3 \mathrm{~mm}$ bunch length is lower than might be anticipated, and warrants further investigation.

\section{ESTIMATION OF MODAL KICK VALUES}

In order to improve beam tracking simulations [9] it is desirable to expand the kick factors out into a set of modes. For cylindrically symmetric geometries the wakepotentials can be greatly simplified by performing a calculation over longitudinal slices of the bunch, taking the wakes as

$$
W_{\perp}=W_{m}(s) r^{m-1} \cos [(m-1) \theta]
$$

where $r$ is the offset from the centre of the collimator and $\theta$ is the angle between 'drive' and 'witness' charges.

GdfidL calculates the wakes associated with a line charge, which does not give a pure dipole response. To 05 Beam Dynamics and Electromagnetic Fields model the dipole response, a PEC plane is placed through the centre of the model, the limit as $r \rightarrow 0$ is observed.

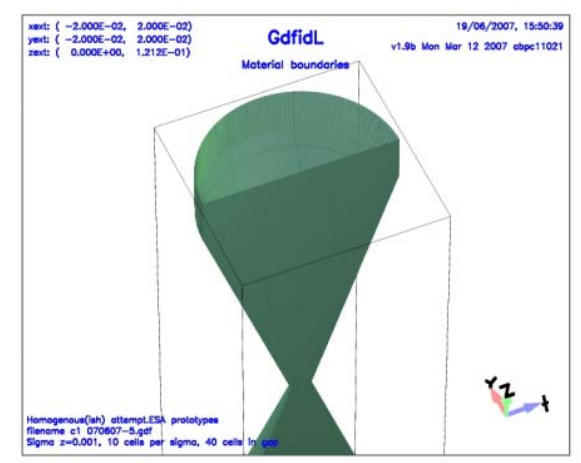

Figure 3: GdfidL geometry for dipole mode, collimator 1. Bunch is displaced in direction of positive $y$.

Similarly, for the quadrupole mode, two planes of electrical boundary can be introduced, the image charges create the quadrupole effect, and the wake as $r \rightarrow 0$ can be approximated to the desired modal value.

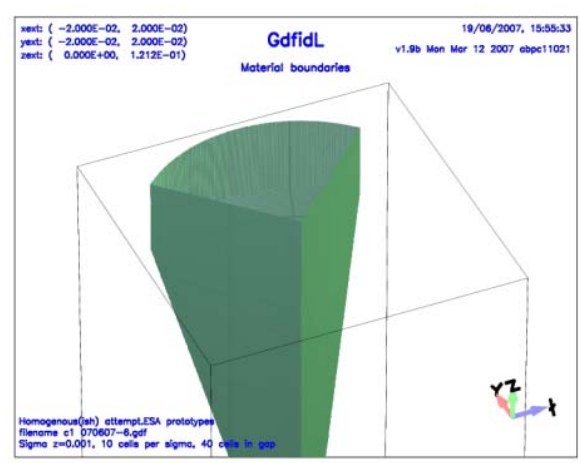

Figure 4: GdfidL geometry for quadrupole mode, collimator 1 . Bunch is displaced in direction of positive $y$.

Some time can be saved by realising that we can still utilize the symmetry in the problem, and GdfidL will handle the drive charge travelling along a magnetic plane to reduce the size of the simulation.

Results for some collimators have been produced, and it is anticipated that tracking codes such as PLACET and MERLIN will soon [8] [9] utilise such models.

\section{COMPARISON WITH OTHER CODES}

There are several codes in existence which perform quasi-2D simulations of cylindrically symmetric collimators, such as ABCI and ECHO-2D. These produce results more quickly than GdfidL, however can not take into account misalignment, or handle rectangular geometry. Nevertheless, these provide a useful means for generation of wakepotentials to be used in PLACET and MERLIN, and it is important to benchmark the codes together. Taking

D04 Instabilities - Processes, Impedances, Countermeasures 
Table 1: Transverse Geometric Kick Factors for 2006 ESA Run Collimators

\begin{tabular}{|c|c|c|c|}
\hline Collimator Number & $\mathbf{0 . 3} \mathbf{m m}(\mathbf{V} / \mathbf{p C} / \mathbf{m m})$ & $\mathbf{0 . 5} \mathbf{m m}(\mathbf{V} / \mathbf{p C} / \mathbf{m m})$ & $\mathbf{1 . 0 m m}(\mathbf{V} / \mathbf{p C} / \mathbf{m m})$ \\
\hline 1 & $1.9 \pm 0.9$ & $1.6 \pm 0.4$ & $1.2 \pm 0.3$ \\
2 & $3.3 \pm 2.3^{*}$ & $2.9 \pm 0.8$ & $2.0 \pm 0.7$ \\
3 & $6.9 \pm 2.6^{*}$ & $5.8 \pm 0.9$ & $4.1 \pm 0.8$ \\
4 & $1.1 \pm 0.3$ & $1.0 \dagger$ & $1.0 \dagger$ \\
5 & $11.1 \dagger$ & $7.9 \pm 0.6 \ddagger$ & $6.1 \pm 1.2 \ddagger$ \\
6 & $3.0 \pm 1.6$ & $2.1 \pm 1.5$ & $1.6 \pm 0.8$ \\
7 & $3.5 \pm 1.1$ & $2.9 \pm 0.5$ & $2.1 \pm 0.6$ \\
8 & $3.0 \pm 2.7$ & $2.4 \pm 0.9$ & $1.6 \pm 0.7$ \\
\hline
\end{tabular}

Table 2: Transverse Geometric Kick Factors for March 2007 ESA Run Collimators

\begin{tabular}{|c|c|c|c|}
\hline Collimator Number & $\mathbf{0 . 3 m m}(\mathbf{V} / \mathbf{p C} / \mathbf{m m})$ & $\mathbf{0 . 5 m m}(\mathbf{V} / \mathbf{p C} / \mathbf{m m})$ & $\mathbf{1 . 0} \mathbf{m m}(\mathbf{V} / \mathbf{p C} / \mathbf{m m})$ \\
\hline 6 & $3.0 \pm 1.6$ & $2.1 \pm 1.5$ & $1.6 \pm 0.8$ \\
$10,11,12$ & $3.7 \pm 1.7$ & $2.6 \pm 1.6$ & $2.0 \pm 0.9$ \\
13,14 & $3.8 \pm 1.3$ & $3.6 \pm 1.0$ & $2.7 \pm 0.8$ \\
15 & $2.4 \pm 1.4$ & $2.5 \pm 1.2$ & $2.0 \pm 1.0$ \\
16 & $2.6 \pm 1.9$ & $2.4 \pm 1.5$ & $1.7 \pm 0.7$ \\
\hline
\end{tabular}

a cylindrical equivalent of collimator 1 described above, simulations were performed with ECHO-2D and GdfidL. The same procedure was performed using the GdfidL results to estimate uncertainties associated with mesh dependence, and the resulting transverse kick factor was $1.367 \pm 0.36 \mathrm{~V} / \mathrm{pC} / \mathrm{mm}$.

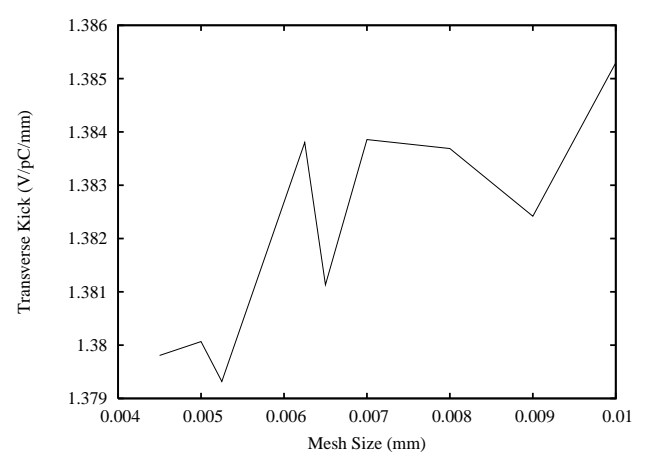

Figure 5: Dependence of ECHO-2D results with mesh for Cylindrical Collimator 1

As can be seen in Figure 5 the echo result of $1.38 \mathrm{~V} / \mathrm{pC} / \mathrm{mm}$ is in extremely good agreement. When it is not necessary to simulate in full $3 \mathrm{D}, \mathrm{ECHO}-2 \mathrm{D}$ can be used to generate results quickly.

\section{CONCLUSION}

There is good agreement between the results for the prototype collimators calculated with ECHO-3D and GdfidL. GdfidL is seen to be a useful design tool for the ILC collimators. Wakefields calculated in GdfidL and similar codes are already being coded into tracking simulations, and more complete simulations of the BDS will soon be 05 Beam Dynamics and Electromagnetic Fields undertaken, taking into account realistic geometries for the spoilers. With further CPU time, the accuracy of calculations can be improved, although there is scope for improvement in understanding the uncertainty, and the calculations are showing agreement with an existing experimental programme.

\section{REFERENCES}

[1] C.D.Beard and J.D.A.Smith, "Numerical Calculations of Collimator Insertions”, EPAC'06, June 2006, Edinburgh, p. 709, http://www.JACoW.org.

[2] C. Beard and R.M. Jones, "Numerical Simulations of Collimator Insertions using MAFIA", EUROTeV-Report-2006103 www.eurotev.org/e158/e1365/e1378/e2317/EUROTeVReport-2006-103.pdf

[3] http://www.gdfidl.de

[4] http://www.ep.ph.bham.ac.uk/cluster/

[5] S. Molloy et al., "Measurements of the Wakefields Due to the Varying Collimator Characteristics", these proceedings.

[6] N.K.Watson et al., "Direct Measurement of Geometric and Resistive Wakefields in Tapered Collimators for the International Linear Collider", EPAC'06, Edinburgh, June 2006, p. 697

[7] I.Zagorodnov and K.L.F.Bane, "Wakefield Calculations for 3D Collimators”, EPAC'06, June 2006, Edinburgh, p. 2859 , http://www.JACoW.org.

[8] A Latina et al., "Wakefield Models for Particle Tracking Codes", these proceedings

[9] A. Bungau and R. Barlow, "Emittance Growth Due to Higher Order Angular Multipole Mode Wakefields in the ILC-BDS Collimators", these proceedings

D04 Instabilities - Processes, Impedances, Countermeasures 